\title{
Grupos de filiación y movilidad poblacional \\ Los chi'bales y la migración de los mayas en el siglo XVIII
}

\author{
Paola Peniche Moreno
}

El siglo XVIII en Yucatán se caracterizó por el intenso flujo migratorio de la población indígena que abandonó sus pueblos de origen para trasladarse a las numerosas fincas españolas que se estaban desarrollando en la península. En el presente artículo se analizan las características esenciales que delineaban al chiłbal o linaje patrilineal, como una de las unidades básicas de organización social de los mayas coloniales, y el papel que éstos desempeñaron en la movilidad poblacional en el siglo XVIII. En este trabajo se parte de la noción de que los desplazamientos no sucedieron de manera aislada, sino que fueron producto de importantes transformaciones políticas, sociales e inclusive territoriales en los lugares de origen y en aquellos de destino. El planteamiento central es que si bien la migración afectó ciertos ámbitos de la organización sociopolítica indígena, contribuyó a la perpetuación de los mecanismos básicos de convivencia nativa, como eran los linajes.

Eighteenth Century Yucatan was marked by intense migration flows of the indigenous population that abandoned its villages of origin and moved to the numerous Spanish fincas, which were developing in the Peninsula. This article analyzes the essential features which outlined chibal or patrilineal lineage as one of the basic units of social organization of the colonial Mayas, and their role in population movements during the 18th Century. This paper is based on the notion that the displacements did not occur in a disconnected way, but rather were the result of important political, social and even territorial transformations both in the place of origin and in that of reception. The central idea is that although migration affected certain spheres of native socio-political organization, it contributed to the perpetration of basic mechanisms of native communal life, such as lineage.

PAOLA PENICHE MORENO: UNAM. 
$\mathrm{E}$ 1 objetivo de este artículo es presentar una reflexión sobre las características esenciales que delineaban al chibal ${ }^{1}$ o linaje patrilineal como una de las unidades básicas de organización social de los mayas coloniales, y el papel que éstas desempeñaron en la movilidad poblacional en el siglo XVIII. En este trabajo se parte de la noción de que los desplazamientos no sucedieron de manera aislada, sino que fueron producto de y trajeron consigo cambios y transformaciones políticas, sociales e inclusive territoriales, tanto en los lugares de origen como en aquéllos de destino. El planteamiento que guía el análisis estriba en que si bien la migración afectó ciertos ámbitos de la organización sociopolítica indígena, también contribuyó a la perpetuación de los mecanismos básicos de convivencia nativa, como eran los linajes patrilineales. $^{2}$

Esta movilidad poblacional estuvo marcada por una fuerte tendencia de los indios a abandonar sus pueblos de origen para remontarse a las fincas ganaderas que se estaban desarrollando y multiplicando en la península. ${ }^{3}$ Tal fenómeno debe ser considerado como migración, en tanto que implicó la adaptación de los sujetos en espacios y entornos diferenciados, la interrupción de actividades en un lugar, así como su reorganización en otro. ${ }^{4}$

\footnotetext{
${ }^{1}$ Ch'ibal: linaje, casta, genealogía por línea recta, Calepino de Motul, vol. I, 1995, p. 262. Ch'ibal: casta, linaje, genealogía por línea recta; de aquí sale: generación o linaje por parte de padre, casta, por linaje generalmente. Diccionario Maya Cordemex, 1980, p. 133.

2 El presente artículo se desprende de una investigación más extensa sobre la migración indígena en el Yucatán del siglo XVIII, sin embargo, el de los linajes quedó como un tema que estuvo lejos de llegar a consideraciones definitivas. Por el momento me encuentro realizando un estudio de mayor alcance y profundidad sobre la organización social y el parentesco entre los mayas de Yucatán, cuyos resultados en un futuro podrán enriquecer los planteamientos que aquí se esbozan. ${ }^{3}$ La importancia del fenómeno de la migración en el siglo XVIII ya ha sido apuntado por varios autores como M.C. García Bernal, La sociedad de Yucatán, 1972, y Desarrollos indígena y ganadero en Yucatán, 1994; N. Farriss, Nueclation versus Dispersal, 1978, La sociedad maya bajo el dominio colonial, 1992; R. Patch, Maya and Spaniard, 1993; R. Solís, Bajo el signo de la compulsión, 2000.

${ }^{4}$ Algunos estudios sobre migraciones contemporáneas coinciden en señalar que para considerar a un movimiento poblacional como un fenómeno de migración, este deberá suponer para el sujeto un cambio de entorno político-administrativo, social y/o cultural relativamente duradero; o, dicho de otro modo, cualquier cambio permanente de residencia que implique la interrupción de actividades en un lugar y su reorganización en otro. Véase C. Blanco, Las migraciones contemporáneas, 2000, p. 17.
}

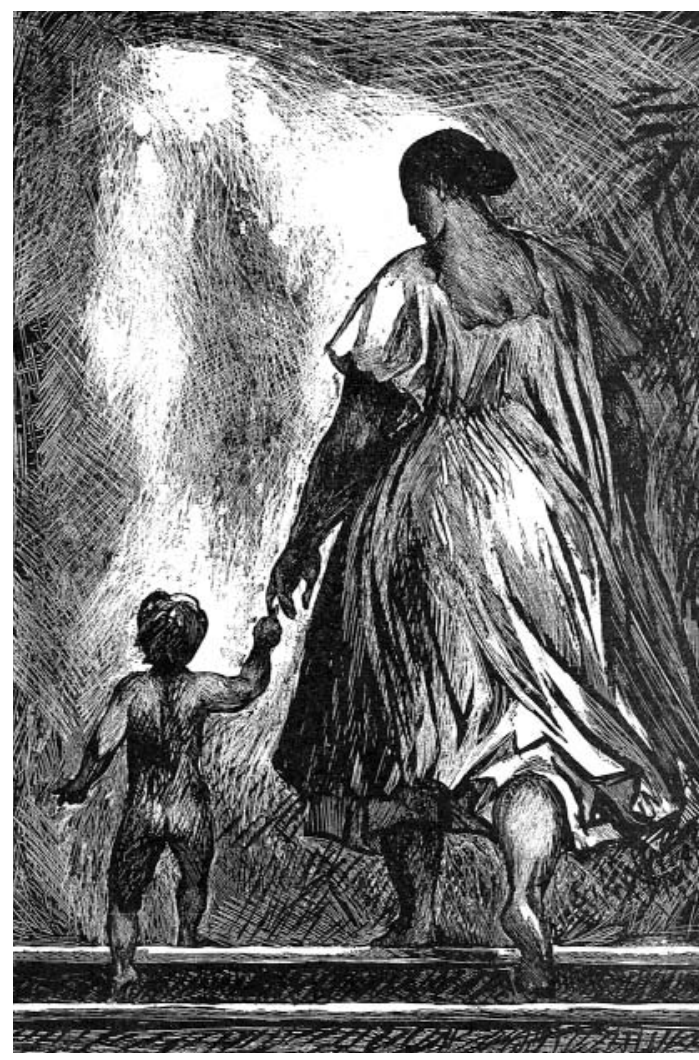

Fernando Castro Pacheco, Pasos de mujer, 1954.

En la época colonial el manejo de recursos estratégicos —principalmente tierra y organización laboral- fueron parte fundamental del sustento del poder político de las autoridades locales y la organización sociopolítica de las repúblicas de indios. No obstante, las relaciones de poder que se establecieron en estos términos, al igual que el manejo de los recursos, estuvieron lejos de ser inmutables, y más bien se vieron inmersas en un proceso de constantes vaivenes que las obligaban a reconfigurarse, adaptarse a situaciones específicas, luchando por sobrevivir o bien, sucumbir lentamente. 5

\footnotetext{
${ }^{5}$ Este artículo se desprende de la tesis de maestría titulada "La diáspora indígena a las estancias yucatecas del siglo XVIII", en donde se analiza el papel que desempeñó la migración en el proceso de debilitamiento de las estructuras políticas de las repúblicas de indios. Paola Peniche Moreno, La diáspora indígena, 2002.
} 


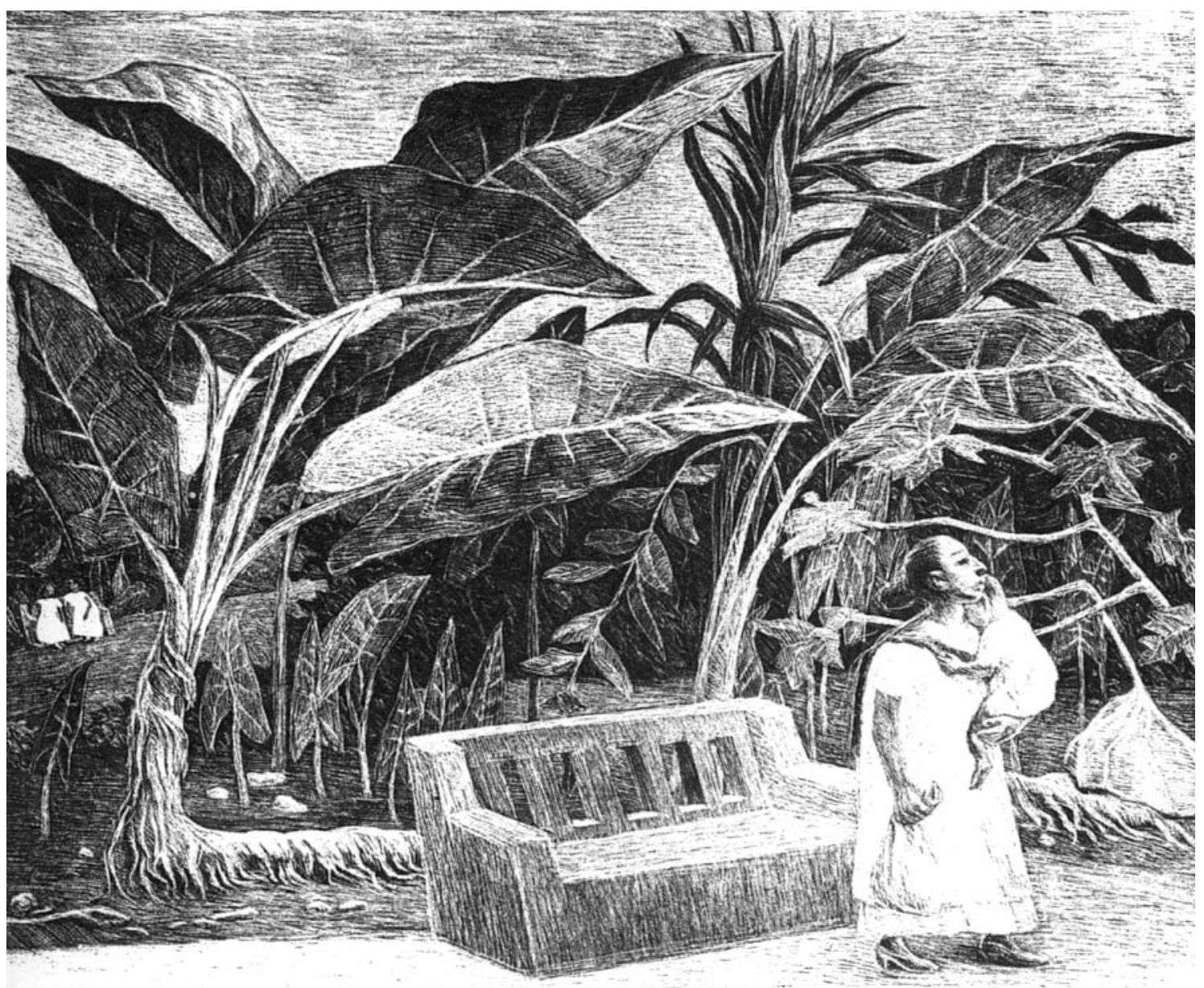

Alfredo Zalce, En la plaza.

El último siglo de la Colonia fue testigo de una serie de pugnas internas que se relacionaron directamente con el manejo de los recursos de las corporaciones indígenas. No fueron pocos los litigios que se establecieron en denuncia de las excesivas cargas económicas y laborales, así como de los malos tratos que sufrían los indios de los pueblos. ${ }^{6}$ Además, hubo una pérdida paulatina tanto de las tierras comunales de cada república como de las que estaban en manos privadas, ya fuera de individuos o gru-

${ }^{6}$ Sobre el trabajo indígena véase R. Solís, Bajo el signo de la compulsión, 2000. pos de filiación. ${ }^{7}$ El detrimento de un control efectivo sobre estos recursos puso en tela de juicio la legitimidad de las autoridades locales, erosionando las bases políticas que sustentaron a las repúblicas de indios. Fue entonces cuando muchos de los actores y los grupos políticos que participaban en las relaciones de poder al interior de las

\footnotetext{
${ }^{7}$ Pedro Bracamonte analiza la problemática territorial en el Yucatán colonial y apunta que en este siglo se registró una importante transferencia de recursos territoriales de los pueblos de indios a empresarios españoles, ya fuera de aquellos que estaban en manos privadas, como aquellos que pertenecían a la coorporación. Pedro Bracamonte, Jurisdicción y tenencia de la tierra entre los mayas de la provincia de Yucatán, 2000, pp. 190-212.
} 
repúblicas exploraron formas alternativas de tener acceso a los recursos que garantizaran su subsistencia, ya fuera como individuos o como grupos.

El siglo XVIII, y especialmente su última mitad, presenció cambios significativos en la economía regional, en donde los sistemas de encomiendas y repartimientos comenzaron a ceder terreno a la propiedad privada como el principal mecanismo de acumulación de riqueza. La ganadería y la agricultura comercial contaron con el apoyo de la Corona, y como consecuencia, las fincas rurales en donde se verificaba tal producción experimentaron una notable expansión. ${ }^{8}$ Este crecimiento fue estimulado además por el crecimiento demográfico que experimentó la población indígena y no indígena en el siglo XVIII, ${ }^{9}$ lo que alentó a muchos españoles a fundar empresas productivas ante el incremento de la demanda de alimentos.

De forma gradual, estas fincas fueron convirtiéndose en una alternativa para los indios que buscaban opciones de vida fuera de sus pueblos de origen, alentados por la necesidad de los empresarios españoles de allegarse de mano de obra. El atractivo más evidente que tuvieron estos centros para los indios que optaban por migrar a ellos era la posibilidad que representaban de evadir, sino todas, una parte importante de las cargas a las que se veían sujetos en sus repúblicas de adscripción, como los tequios, los repartimientos y los servicios personales. Pero otros factores seguramente influyeron en la decisión de los indios de aventurarse en la empresa migratoria, y uno de ellos fue precisamente que las estancias significaron no sólo la oportunidad de evadir cargas y trabajos, sino de

\footnotetext{
${ }^{8}$ Robert Patch sostiene que en el siglo XVIII, especialmente en su última mitad, se puede observar el giro de una sociedad colonial a otra neocolonial, en donde la propiedad privada comenzó a ser el principal mecanismo de acumulación de riqueza, y la "inferioridad" india en relación con los españoles se definía en términos de relaciones de propiedad más que de relaciones coloniales. En este proceso, según argumenta el autor, dos desarrollos fueron importantes: por un lado, el crecimiento de las estancias y haciendas, y por el otro, la decadencia de la encomienda y el repartimiento. R. Patch, Maya and Spaniard..., 1993, pp. 137-138.

${ }^{9}$ Cook y Borah han calculado que en el siglo XVIII la población india en Yucatán aumentó de 127000 en 1736 a 245000 en 1794, es decir casi en un 93\%; el incremento de la población no india, según los mismos cálculos, es aún más significativo, pues en los mismos años aumentó en un 202.94\%, de 34000 a 103000 habitantes. S. Cook y Woodrow Borah, Ensayos sobre historia de la población, t. 2, p. 119.
}

negar al mismo tiempo su pertenencia a determinada república, así como tener acceso por nuevos caminos a los recursos necesarios para su reproducción física y social.

En este sentido, el abandono de los pueblos y el traslado de los indios a las estancias significó mucho más que un desplazamiento espacial, fue principalmente social y cultural en su más amplia expresión. La diáspora maya a las estancias coloniales trajo consigo transformaciones importantes, sobre todo porque los lazos que vinculaban a esta población a la tierra y a la organización del trabajo colectivo dejaron de formar parte integral de la vida corporativa de los pueblos de indios y comenzaron a reestructurarse en el ámbito de la esfera privada de la producción. Sin embargo, es posible observar que el tipo de relaciones que establecieron los indios que se adscribieron al trabajo de las estancias se edificaron sobre antiguos mecanismos de organización, y uno de ellos fue precisamente el de los linajes patrilineales.

De esta manera, la movilidad poblacional estuvo estrechamente relacionada con el inicio de un proceso de desestructuración de los pueblos coloniales como entidades corporativas, puesto que implicó la pérdida de lazos de la población indígena migrante con sus comunidades de origen, cuestionando y, a la vez, debilitando la legitimidad de la dirigencia nativa y el funcionamiento interno de las repúblicas de indios. Pero al mismo tiempo, la migración contribuyó al crecimiento de las fincas españolas y a su desarrollo como importantes centros de producción y poblamiento. Así, el problema de la movilidad de la población maya en este periodo se convirtió en un fenómeno que trastocó algunas de las formas de organización que habían funcionado como las bases de articulación de la sociedad indígena al orden colonial: el de las repúblicas de indios.

Sin embargo, como se verá más adelante, el campo político de la sociedad indígena era mucho más complejo que la sola formación de repúblicas de indios con cabildos que administraban tanto la población como los recursos de los pueblos. Al interior de estos existían facciones que desempeñaban el papel de organizadores de la vida política y luchaban por su permanencia - como los ch'ibales-, y cuando los pueblos no les proporcionaban la oportunidad de participación en el ejercicio 
del poder que les asegurara la conservación de determinados espacios, optaban por desaparecer de la arena política, rompiendo los lazos que los ligaban a la república y buscaban en otros sitios las condiciones propicias para su preservación.

En este sentido, podríamos entender los desplazamientos poblacionales, con el concomitante abandono de las repúblicas de indios, como un elemento de carácter político, como causa, y al mismo tiempo resultado, de un faccionalismo al interior de las repúblicas de indios, donde los grupos que la conformaban - como los linajesse escindían de la unidad política mayor, separación que se expresó de manera evidente en la migración. De ahí que distinguir los aspectos esenciales del chibal como unidad de organización nos permitirá determinar en qué medida la migración no implicó su desestructuración, a pesar de que su adscripción a los pueblos sí se haya transformado. Por otro lado, también servirá para analizar los cambios y continuidades sufridos por los mayas que se adscribieron al servicio de las estancias.

\section{LOS CHI'BALES EN EL UNIVERSO DE LA ORGANIZACIÓN SOCIOPOLÍTICA DE LOS MAYAS}

La historiografía sobre la región que ha analizado la organización sociopolítica de los mayas antes de la conquista coincide en señalar la existencia de, al menos, cuatro diferentes niveles de articulación más allá de la familia extensa: 1) los ch'ibales, que conformaban grupos de filiación que, pese a que el parentesco era una de sus características, trascendían el entorno familiar para formar linajes que se insertaban en distintos ámbitos como el de tenencia de la tierra o el político; 2) los cuchteeles, que en la época colonial se denominaron "barrios" o "parcialidades -aunque este último término es más complejo y se aplica a otras entidades políticas - que conformaban también unidades políticas con características de jurisdicción territorial y sujeción personal; 3) el pueblo o cah, nivel sobre el cual los españoles construyeron la base que impulsó la colonización, a través de las congregaciones y la imposición de instituciones como las repúblicas de indios y el cabildo; y 4) las provincias o cuchcabales, que en la época previa al contacto conformaron las unidades más complejas de organización política y territorial.

En la base de la organización social de los mayas, tanto en la época prehispánica como colonial, se encontraba la familia extensa. Los autores que han investigado el tema ${ }^{10}$ coinciden en señalar la importancia de esta unidad de organización como una de las entidades que aglutinaba a los individuos en torno a determinados objetivos, creando redes de interdependencia con derechos y obligaciones mutuas. La historiografía sobre la región ha identificado a estas unidades como parte del complejo de organización política de los mayas, apuntando que se trataba de grupos de varones emparentados patrilinealmente, con sus mujeres e hijas solteras, que desplegaban lazos de solidaridad e involucraban a todos los individuos que compartían el mismo patronímico. ${ }^{11}$ Asimismo, se ha señalado que las familias extensas funcionaban como una unidad económica cooperativa, integrando grupos residenciales de carácter patrilocal, aunque la colonización, con sus políticas de aculturación, favoreció e inclusive impuso la residencia por grupos de familias nucleares. ${ }^{12}$

Sin embargo, estudios recientes han señalado que la organización de los grupos de parentesco era mucho más compleja. Así, se ha identificado otra unidad de organización que, a pesar de compartir las características asignadas a la familia extensa, la trasciende. Aun cuando el parentesco era una de las características principales de estas células de organización, al parecer no era la única. Por ello, para su análisis en este trabajo se ha decidido recurrir al término de filiación en vez del de parentesco, puesto que la consanguinidad no bastaba por sí misma para reproducir los vínculos que cohesionaban estas unidades. De tal forma, la filiación nos permite conectar dos campos, el parental y el político, mientras que el parentesco nos limita al ámbito de las relaciones consanguíneas. ${ }^{13}$

\footnotetext{
${ }_{10}$ R. Roys, The Indian Bakground..., 1943; T. Okoshi, Los canules: análisis etnohistórico del Códice de Calkiní, 1992; N. Farriss, La sociedad maya bajo el dominio colonial, 1992; P. Bracamonte, Jurisdicción $y$ tenencia de la tierra..., 2000; M. Restall, The maya World..., 1997. ${ }_{11}$ R. Roys, The Indian Bakground, op. cit., p. 33.

${ }^{12} \mathrm{~N}$. Farriss, La sociedad maya bajo el dominio colonial, op. cit., p. 216.

${ }^{13}$ Tal es el planteamiento de Meyer Fortes, quien concibe al concepto de filiación como aquel que conecta al aspecto externo con el interno
} 
Mathew Restall atribuye a esta unidad que trasciende a la familia extensa características propias tanto de linajes como de clanes exogámicos y, por tanto, concluye que el mejor término para denominar esta forma de organización es su nombre en maya: ch'ibal. ${ }^{14}$ No obstante, la literatura antropológica que aborda las formas de organización social basadas en el parentesco demuestra que, en realidad, eran pocas las características que los ch'ibales mayas de la época colonial compartían con los clanes, y más los rasgos que nos permiten tratarlos como linajes agnaticios. ${ }^{15}$

El principal argumento que nos aleja de concebir a los chi'bales como una forma de organización clánica es que ésta se basa en unidades que se componen de varios linajes en los que se supone una filiación común, pero que no se demuestra necesariamente, ${ }^{16}$ y se aplica esta categoría a gente dispersa que no forma grupos corporativos y que en la mayoría de los casos rebasa el ámbito local. ${ }^{17}$ Si bien es probable que en la época prehispánica y quizá en inicios de la Colonia la organización de los mayas haya trascendido las fronteras de los linajes para formar parte de una entidad mayor basada en clanes, ${ }^{18}$ no contamos con evidencias que nos permitan suponer que, si lo fueron, los clanes prehispánicos hayan subsistido en los siglos posteriores, ni que los grupos de filiación como unidades de organización hayan trascendido el ámbito local. El análisis de los papeles de los Xiu de Yaxá realizado por Tsubasa Okoshi, por ejemplo, revela cómo la colonización restringió el alcance del linaje Xiu, especialmente después de la muerte del último halach uinic, cuando cada una de sus ramas formó su propio destino para establecerse en diferentes pueblos. ${ }^{19}$

o de consanguinidad. En L. Dumont, Introducción a dos teorías de la antropología social, 1975, pp. 76-78.

${ }^{14}$ M. Restall, op. cit., p. 17.

${ }^{15}$ Linajes trazados por vía masculina.

${ }^{16}$ R. Fox, Sistemas de parentesco y matrimonio, 1967, p. 46.

17 J. Middleton y D. Tait, "The Lineage and the Lineage System”, en Kingship and Social Organization, 1968, p. 155.

${ }^{18}$ De hecho, hay autores que han apuntado la posibilidad de que la organización de los individuos por grupos de patronímicos haya sentado las bases para la existencia de la organización de las provincias, ampliando definitivamente al alcance de la organización de los ch'ibales. M. Restall, op. cit., p. 28; R. Patch, Maya and Spaniard..., op. cit., p. 65. 19 T. Okoshi, "Los xiu del siglo XVI", Mesoamérica, vol. 21, núm. 39, 2000 , p. 230.
Al parecer, los ch'ibales en la época colonial conformaban grupos locales de descendencia unilineal que, a través del vínculo masculino, compartían el mismo apelativo y constituían por lo tanto grupos de filiación patrilineal. Al igual que en cualquier grupo con esta clase de filiación, ${ }^{20}$ los ch'ibales eran de tipo exogámico. Por ello, los varones del grupo debían buscar consortes en otro linaje, al tiempo que debían exportar hermanas e hijas. De hecho, la historiografía de la región ha observado que la migración que se registró entre repúblicas de indios estuvo estrechamente relacionada con la característica exogámica de los ch'ibales, ya que se sugiere que el matrimonio pudo haber sido la principal causa de la movilidad de los forasteros. ${ }^{21}$

Por otro lado, entre los grupos de filiación existe una tendencia a conformar unidades políticas, en las que los valores de linaje y las relaciones de parentesco se constituyen en relaciones políticas en determinadas situaciones, sin querer decir con ello que los grupos políticos y los de linaje sean del todo idénticos, sino que existe en cierto modo una correspondencia entre ellos. ${ }^{22}$ En este sentido, Mathew Restall ha señalado la posibilidad de que la organización de los ch'ibales haya creado entidades mayores que, a manera de parcialidades, conformaban subdivisiones que se integraban con facciones sociales y políticas al interior de los pueblos. Sin embargo, la mayoría de los estudios que han abordado esta unidad proponen no confundirla con subunidades geopolíticas, a la manera como se ha visto a los calpulli del centro de México. ${ }^{24}$ De hecho, las posturas que han interpretado a la organización de los antiguos mexicanos con carácter básicamente parental han sido trascendidas por otros estudios, como el de Luis Reyes García, quien concibe al calpulli como una entidad dinámica en donde el parentesco es sólo una de las cualidades, entrelazada con otras condiciones de índole de jurisdicción territorial, de grupo étnico, sujeción, tenencia de la tierra y culto. ${ }^{25}$ De manera

\footnotetext{
${ }^{20}$ R. Fox, op. cit., p. 88.

${ }^{21}$ P. Bracamonte y G. Solís, op. cit., p. 126.

22 Evans-Pritchard, Los Nuer, 1977, p. 213.

${ }^{23}$ M. Restall, op. cit., p. 29.

${ }^{24}$ M. Restall, op. cit.; P. Bracamonte y G. Solís, op. cit.

${ }^{25}$ L. Reyes García, "El término calpulli en documentos del siglo XVI", en Documentos nahuas de la ciudad de México del siglo XVI, 1996, pp. 35-56.
} 


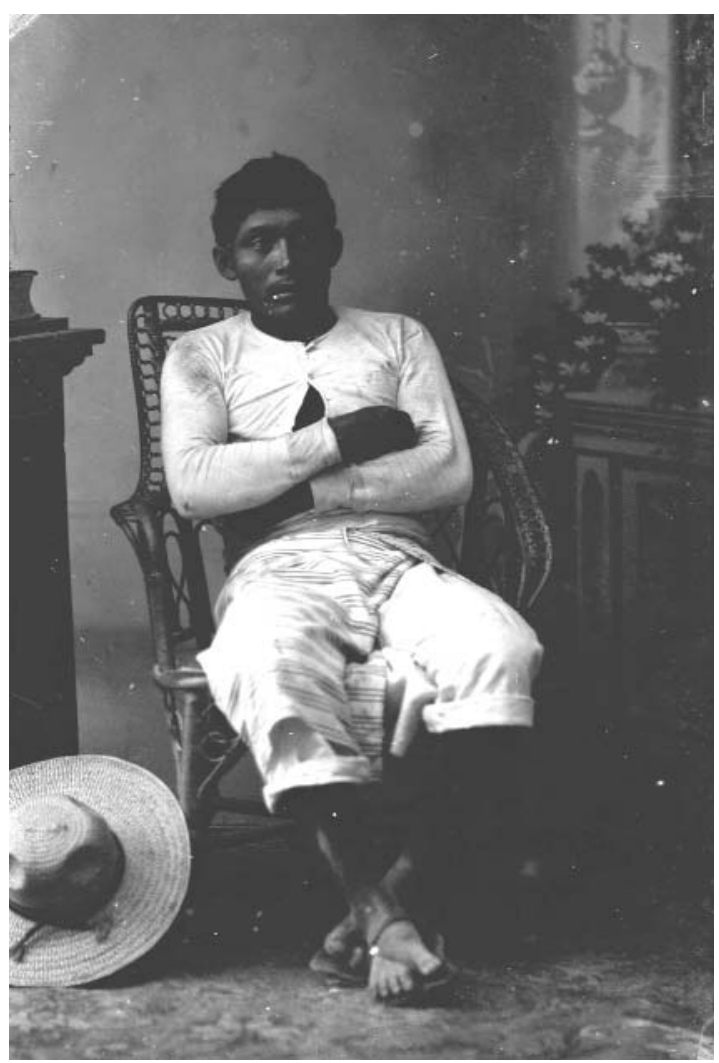

Retrato de campesino en estudio, 1915 / Fototeca Pedro Guerra, Universidad Autónoma de Yucatán.

similar, Mathew Restall planteó que para el caso yucateco la articulación de los individuos a las unidades de los ch'ibales se daba obviamente por medio del parentesco, aunque también por la propiedad de la tierra. ${ }^{26}$ De hecho, los estudios antropológicos clásicos sobre linajes nos revelan que incluso en las sociedades sin instituciones políticas, donde el parentesco cobra aún mayor importancia en la organización social, éste no resulta ser la única característica que delimita a estos grupos ni siempre la más importante. ${ }^{27}$

El análisis de la tenencia indígena de la tierra en la época colonial realizado por Pedro Bracamonte ha sacado a

${ }^{26}$ M. Restall, op. cit., p. 17.

${ }^{27}$ Evans-Pritchard, op. cit., p. 178. la luz nuevas aportaciones relativas al papel que desempeñaron estas unidades de organización, puesto que señala la posibilidad de que el ch'ibal dominante en una parcialidad agrupara a un número de personas, ejerciendo un dominio sobre una fracción de tierra y exigiendo por su uso renta y servicios. Así, los grupos de ch'ibales conformarían al interior de las repúblicas de indios facciones políticas que competían por el poder. De acuerdo con este autor, la tierra de los ch'ibales era determinante para explicar la estratificación, los vínculos de sujeción y el acceso restringido al poder político. ${ }^{28}$ Con esta mirada es posible pensar que, al estar las facciones políticas al interior del cah asociadas a grupos de linajes, todas las personas de la comunidad que no fueran miembros de un grupo quedaran asimiladas a uno de ellos a través de relaciones políticas y que por esa razón se expresaban con frecuencia en términos de valores del linaje.

Cabe señalar que a través de los chỉibales los individuos se enlazaban en primera instancia con el pueblo. En la república de nacimiento la pertenencia a un grupo de filiación determinaba el acceso del individuo a ciertos derechos y lo obligaba a cumplir con determinadas cargas. ${ }^{29}$ De esta forma, estos grupos funcionaban como las unidades políticas básicas dentro de la jurisdicción del cah o pueblo. Sin embargo, permanece aún la interrogante, advertida por Roys, sobre la existencia de una jerarquización entre los integrantes de los ch'ibales, pues a pesar de que un chibal contara entre sus miembros con individuos que pertenecieran a la nobleza y ejercieran el poder, los beneficios económicos o políticos no llegaban por igual a todos los miembros de un patrilinaje. ${ }^{30}$

No obstante, este acceso diferenciado a los recursos y a los distintos estratos sociales y de ejercicio del poder se puede explicar por el principio de segmentación inherente a los sistemas de linaje. Esto es, que los linajes tienden a subdividirse en grupos más pequeños —en ocasiones familias extensas-, cada uno con genealogías de menor extensión, formando unidades en un sistema de segmentos. Tal principio perpetúa el sistema patrilineal, pues

\footnotetext{
${ }^{28}$ P. Bracamonte, Jurisdicción y tenencia de la tierra..., op. cit., p. 121.

${ }^{29}$ Bracamonte y Solís, op. cit., p. 127.

${ }^{30}$ Roys, The Indian Background..., op. cit., pp. 35-36.
} 
cada hombre aparece como fundador potencial de un linaje. ${ }^{31}$ El sistema segmentario que define a los linajes determina entonces la posición de sus miembros. ${ }^{32}$ Es decir, que en tanto unos segmentos del mismo linaje tenían acceso al poder, otros componían el grueso de la población macehual. De esta manera, la distancia social entre los grupos puede ser concebida como la distancia social existente entre las unidades segmentadas del sistema de linajes.

Dicho principio de segmentación nos presenta al sistema político como uno en equilibrio, integrado por fuerzas opuestas, unas que apuntan hacia la escisión y otras a la fusión, oscilando entre la propensión de todos los grupos a segmentarse y su tendencia a combinarse con otros segmentos del mismo orden. En un sistema donde los grupos tienden a dividirse en partes opuestas y donde dichas partes se funden en relación con otros grupos, la totalidad aparece como unidad en un contexto y se muestra segmentada en otro. Los linajes tienen la característica de existir en un estado de continua segmentación y oposición complementaria. ${ }^{33}$

Tener presente el principio de segmentación de los linajes resulta de suma importancia para abordar diversos aspectos de la organización de un grupo, tales como el ritual, el ámbito económico o la vida política. Cuanto más próximo se halle un segmento del linaje principal, mayor será su prelación en cuestiones rituales, económicas y/o políticas. ${ }^{34}$ Pero también, como se verá en el siguiente apartado, la segmentación de los sistemas patrilineales nos permitirá abordar los principales patrones de migración, y de esta forma determinar en qué grado operaron los cambios en la organización social que trajo consigo la movilidad geográfica.

\section{LOS DESPLAZAMIENTOSY LA SEGMENTACIÓN DE LOS CH'IBALES}

Como se señaló en un principio, la migración implicó la pérdida de lazos de un sector de la población indígena

${ }^{31}$ J. Middelton y D.Tait, op. cit., p. 155.

32 Evans Pritchard, op. cit., p. 16.

33 Ibidem, p. 166.

${ }^{34}$ R. Fox, op. cit., p. 117. con sus comunidades de origen, debilitó en consecuencia el funcionamiento de los pueblos coloniales como entidades corporativas y, al mismo tiempo, cuestionó la legitimidad de la dirigencia nativa. No obstante, en este trabajo se plantea que la incorporación de contingentes importantes de población maya al trabajo de las empresas españolas no significó una transformación radical en las formas de convivencia nativa, sino que brindó a un sector de la población indígena la posibilidad de mantener importantes continuidades en algunos de sus principios organizativos.

Es necesario, por tanto, evaluar los cambios y continuidades que se experimentaron en la base de la organización social encarnada en los ch'ibales, al tiempo que se rompían los vínculos y lazos de sujeción que los ligaban a las entidades políticas mayores o repúblicas de indios. La pregunta central que guía el análisis y la reflexión es en qué medida la migración a las estancias afectó esas unidades.

Para ello, partimos de la necesidad de abordar los principales patrones migratorios que nos permitan observar la manera como los indios se reagruparon en las fincas rurales. Las fuentes de información empírica más importantes para analizar tales patrones son las matrículas de tributarios que formaron tanto funcionarios civiles como eclesiásticos, en las que registraban por nombre y apelativo a todos los residentes tanto de los pueblos como de las estancias, ranchos y sitios que se encontraban en la provincia. En especial, nos hemos basado en las matrículas que en 1803 formara el intendente Bernardo Bruno como resultado de la visita que llevó a cabo en 11 pueblos de la provincia, para realizar un informe sobre el tributo de 13 reales anuales, mitad por San Juan y mitad por Navidad, además del Servicio Real que se tasaba a medio real por cada indio. ${ }^{35}$

De las distintas subdelegaciones en las que estaban distribuidos los 11 pueblos que fueron objeto de la visita del Intendente, se preferenció la de Izamal puesto que era la que presentaba más concentración de pueblos. De ahí, se seleccionaron los pueblos de Izamal, Cacalchén,

35 AGN, Tributos, 1803, vol. 64, 65, 66 y 67. 


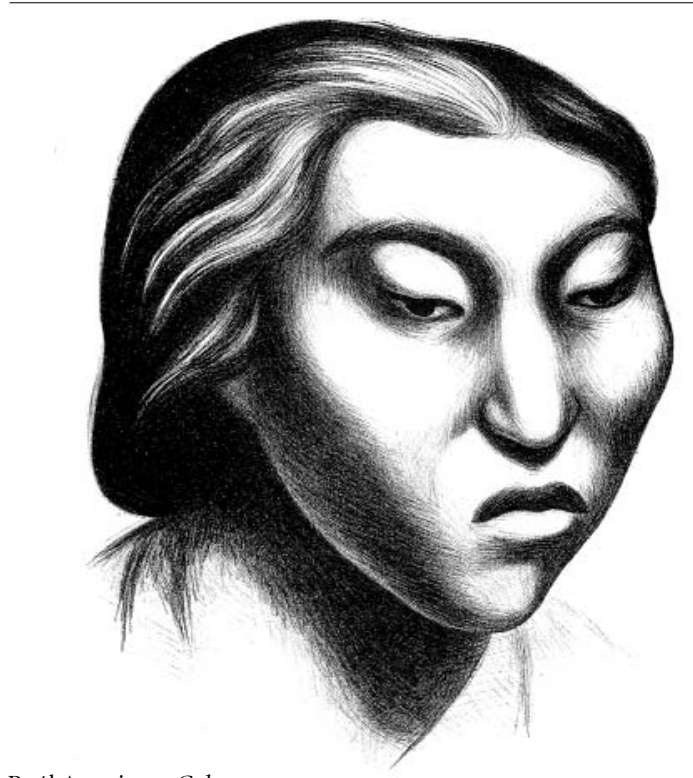

Raúl Anguiano, Cabeza maya, 1949.

Bokobá y Zitilpech, que independientemente de la cercanía que existía entre uno y otro, y a pesar de que formaban parte de la misma subdivisión política, reflejan tanto constantes como variaciones de un mismo fenómeno. Asimismo, se aislaron algunas variables, tales como el patronímico de los indios, sexo, lugar de residencia y origen o procedencia de los mismos, todas ellas en dos esferas, las cuales nos permitieron abordar el fenómeno migratorio en su conjunto: los puntos de origen y destino de los desplazamientos.

Cabe recordar la manera como los sistemas de linajes patrilineales se definen en función de un principio segmentario, en donde las unidades que lo conforman aparecen como una totalidad en un contexto, y como partes opuestas en otro. Sin embargo, a pesar de la escisión de los linajes, las unidades menores conservan su pertenencia e identificación como integrantes de un mismo linaje. ${ }^{36}$

A partir de tales principios se aborda ahora la migración, así como también el papel que desempeñaron las formas de organización indígena en este proceso. Analizar los principales patrones de segmentación de los linajes a partir de los cambios de residencia de los portadores de determinado patronímico nos permitirá dar indicios de la

${ }^{36}$ Fox, Sistemas de parentesco y matrimonio, op. cit., p. 168. persistencia de tales unidades de organización, pues partimos de la hipótesis de que si bien los desplazamientos indígenas hacia las fincas rurales trajeron consigo transformaciones importantes, no siempre implicaron la desaparición de las formas básicas de articulación social, sino que la migración refleja el principio básico de segmentación inherente a los sistemas de linajes patrilineales y, por lo tanto, su persistencia. Sin embargo, es importante señalar que en la muestra elegida es posible encontrar variaciones importantes, pues algunos pueblos y sus estancias reflejan una clara tendencia a mantenerse como unidades de linaje, en tanto otros presentan una mayor disolución.

Los ejemplos de ch'ibales que conservaron los principios básicos de su organización a pesar de la migración, provienen en su mayoría de los registros de la población originaria de la jurisdicción de los pueblos de Cacalchén y su visita parroquial Bokobá. En ambos casos es posible observar cómo un alto porcentaje de los indios que abandonaron sus pueblos de origen lo hicieron colectivamente, en el marco de unidades de linaje que se desprendieron de uno mayor.

A pesar de que la proporción entre hombres y mujeres que habitaban en estancias de ambos pueblos es muy similar, cerca del $75 \%$ de los hombres se agruparon por segmentos de ch'ibales con más de diez integrantes, en tanto la variación en los apelativos era proporcionada por las mujeres. Esto no significa que las mujeres tendieran más a la migración individual, puesto que a través del matrimonio formaban parte de los ch'ibales mayoritarios, pues cabe recordar que se trataba de linajes que privilegiaban la línea masculina.

En las estancias de la jurisdicción de Cacalchén, por ejemplo, se identifican con regularidad dos apelativos (Mukul y Couoh) que, además de integrar a la mayoría de la población que habitaba en las estancias, presentan una segmentación tendiente a reproducir las formas de agrupación patrilineal por unidades menores que se desprendieron de un linaje máximo o quizá un linaje mayor. ${ }^{37}$ La migración a las estancias reforzó su escisión,

${ }^{37}$ Evans Pritchard, quien analiza el principio de segmentación entre los linajes patrilineales de los Nuer, establece distintas categorías que le permiten dar seguimiento a la historia de segmentación de estas unidades de organización social y política, así como distinguir las situaciones 


\section{GRÁFICO 1. Distribución de la población migrante según género y ch'ibal que reside en las estancias de la jurisdicción de dos pueblos}

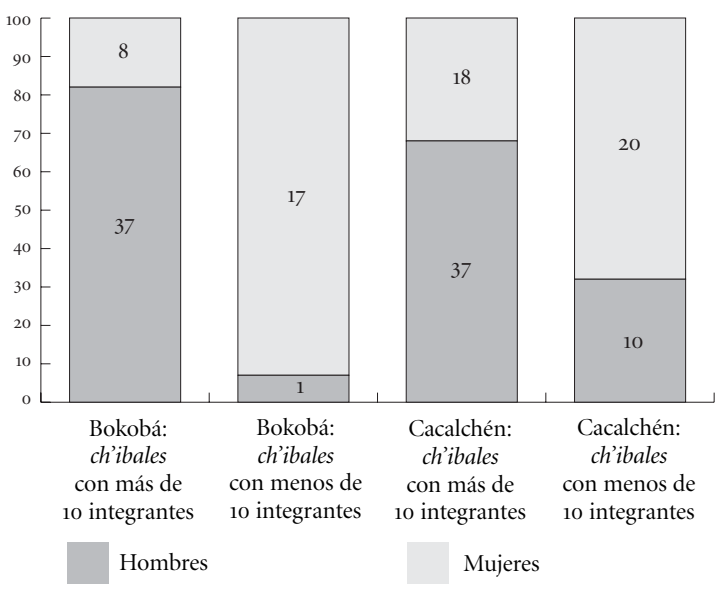

FUENTE: AGN, ramos 16 Tributos, vols. 64, 65, 66 y 67.

pero reagrupándolos en unidades mínimas que privilegiaron la asociación por vía masculina.

Sin embargo, no podemos saber hasta qué punto la escisión se llevó en el momento que optaron por migrar, o si ya conformaban unidades segmentadas en sus lugares de origen y como tales se desplazaron, puesto que no sabemos la temporalidad precisa en que estos individuos abandonaron sus lugares de origen para remontarse a las fincas. Si tuviéramos tales datos podríamos saber si quien migró fue tan sólo un hombre, y como tal fundó en su nuevo lugar de residencia un segmento de su linaje de adscripción, o fue todo el grupo en su conjunto. Aun si se tratara del primero de los casos, nuestra hipótesis central no se vería del todo alterada ya que, como hemos discutido con anterioridad, el hecho de que los hijos de un individuo hayan nacido lejos del núcleo parental original no impide que sigan siendo reconocidos como parientes e integrantes de un mismo ch'ibal.

específicas en que tales segmentos se unen o actúan como partes contrapuestas. Así, distingue entre linajes máximos, que son los segmentos mayores en que se divide el clan; linajes mayores, para referir a los segmentos en que se divide un linaje máximo; linajes menores, que son los segmentos en que se divide un linaje mayor, y linajes mínimos, para hacer mención a las unidades que se desprenden de este último. E. Pritchard, Los Nuer, op. cit., p. 20.
GRÁFICO 2. Origen de los indios mayores de 14 años que residen en las estancias de la jurisdicción de Cacalchén

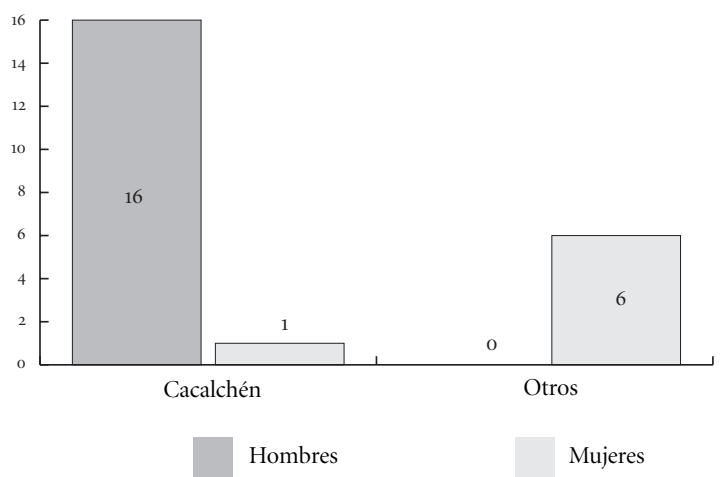

FUENTE: AGN, ramos 16 Tributos, vols. 64, 65, 66 y 67.

Las estancias de la jurisdicción de Bokobá nos presentan una situación un tanto distinta a las de Cacalchén, puesto que en total suman solamente cuatro fincas y, por lo tanto, el número total de individuos que residieron en ellas fue menor. Este hecho podría significar dos cosas distintas: ya sea que los indios de Bokobá no hubieran migrado o bien que lo hayan hecho a otros lugares. Las evidencias apuntan a esta última posibilidad, pues mientras las fincas de Bokobá albergaron a 15 indios originarios de la misma cabecera, las de la jurisdicción de Izamal llegaron a registrar hasta 46 indios mayores de 14 años provenientes de ese pueblo. Es posible que esta disparidad se debiera en parte a que la opción de migrar a las estancias del mismo pueblo hayan sido menores por el escaso número de fincas que se hallaban en su jurisdicción y, también, porque seguramente existía toda un serie de restricciones relacionadas con la producción y la cantidad de tierras disponibles. También influyó probablemente la capacidad de estas fincas de albergar a un número mayor de migrantes, pues mientras que en otros lugares llegaron a habitar hasta 100 indios en las estancias, en Bokobá la que mayor concentración de población presentó era de 13 habitantes.

Aun así es posible identificar en estos asentamientos una distribución de apelativos que refleja una tendencia a dibujar la reagrupación de los migrantes en segmentos de linaje, en donde la asociación de varones portadores del 


\section{GRÁFICO 3. Proporción de los indios forasteros mayores de 14 años que residen en la jurisdicción de Zitilpech}

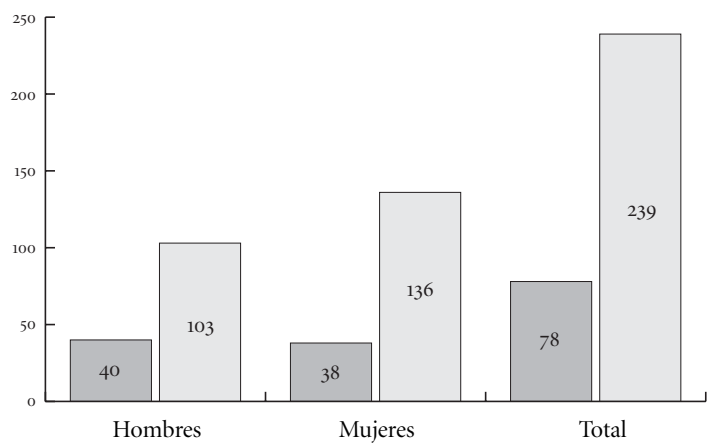

Locales

Forasteros

FUENTE: AGN, ramos 16 Tributos, vols. 64, 65, 66 y 67.

mismo patronímico aparece con relativa claridad. Pero es importante señalar que, mientras las subunidades de linaje asentadas en las fincas de Cacalchén eran segmentos de un grupo mayor que aún se conservaba en la ca-

\section{GRÁFICO 4. Distribución de la población mayor de 14 años por ch'ibales y que residen en el pueblo y las estancias de la jurisdicción de Zitilpech}

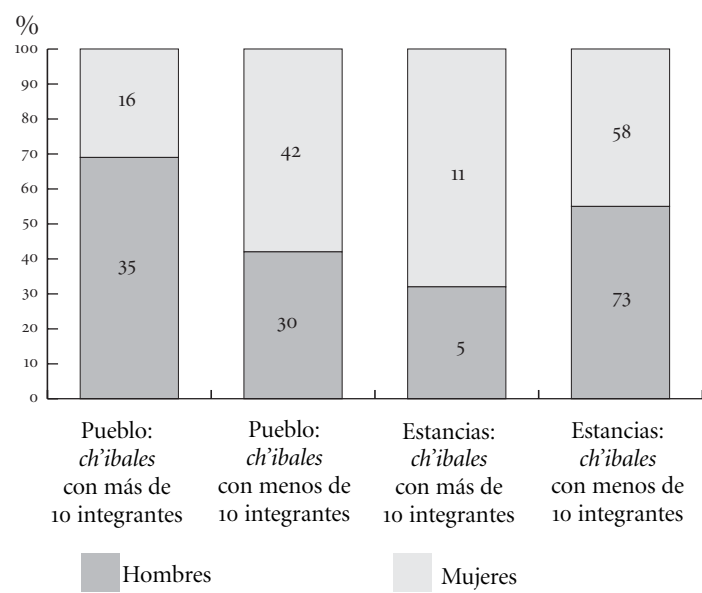

FUENTE: AGN, ramos 16 Tributos, vols. 64, 65, 66 y 67.

tenidos en las matrículas no es posible aseverar que tales ch'ibales se encontraran en un estado de desarticulación puesto que existe la probabilidad de que varones formando unidades de linaje se hayan asentado en las fincas de otros pueblos.

Cabe apuntar que de un total de 145 indios que radicaban en las estancias de Zitilpech, tan sólo cuatro aparecen como originarios de la cabecera de la misma jurisdicción.

Este hecho no debe sorprendernos si tomamos en cuenta que la jurisdicción de este pueblo era una zona receptora de migrantes de muy variados lugares, ya que no sólo en las estancias se registra este fenómeno pues el $40 \%$ de los indios que vivían en el mismo pueblo eran forasteros. Aun así, resulta relevante que en la cabecera de Zitilpech fueran tan sólo cuatro linajes con más de diez miembros los que aglutinaran a cerca de la mitad de la población. Es posible suponer que los ch'ibales con mayor prelación que vivían "bajo campana", no sufrieron un proceso de segmentación semejante al de los linajes de Cacalchén y Bokobá; o que si tal escisión existía al interior del pueblo, ésta no se expresaba en la migración.

Por lo tanto, al referirnos al proceso de fisión de los linajes observable en las matrículas de las estancias de Zitilpech, es importante apuntar que no se trataba de una
${ }^{38}$ Fox establece una diferencia entre segmentación y fisión, pues la primera se refiere a una ruptura total, en tanto que la segunda constituye una forma inherente a los modos de organización de los linajes. R. Fox, op. cit., p. 20. 


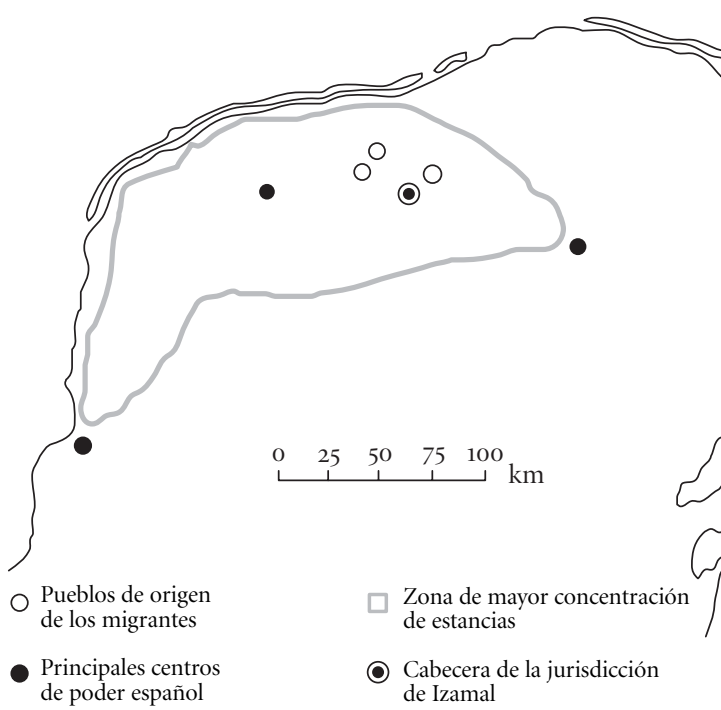

FUENTE: José A. Güémez Pineda, Los mayas ante la emergencia del municipio, 2001, p. 53 (modificado).

descomposición de los ch'ibales originarios de la misma jurisdicción, sino de los de otros pueblos que se desplazaron a estas fincas.

\section{LOS CH'IBALES Y LA RECONFORMACIÓN DEL ESPACIO}

Hasta ahora hemos centrado nuestra atención en la reagrupación de los patronímicos mayas que fueron registrados en las matrículas de 1803 y se ha visto la posibilidad de identificar el proceso de segmentación de los ch'ibales, descrito como un principio inherente a la organización de los linajes. Sin embargo, con base en las mismas fuentes, se debe evaluar la manera cómo tales segmentos y subunidades de los ch'ibales fueron reconformando el espacio rural — particularmente las fincas yucatecas - desplazando nuestro foco de interés a los puntos de destino de la migración. Para ello, hemos optado por tomar como ejemplo las estancias que reflejan tendencias de agrupación disímiles (como las que líneas antes se señalaron entre Zitilpech y las demás jurisdicciones), aunque el resultado del análisis no sea del todo contrastante.
En las fincas que se encontraban en la jurisdicción de Cacalchén se puede observar una marcada tendencia de los indios a mantener en sus nuevos lugares de residencia la organización de los ch'ibales. La estancia Iluch, por ejemplo, fue poblada en su totalidad por un sólo segmento del ch'ibal Pech. El sitio Xan, de la misma jurisdicción, presenta una situación similar a la anterior, aunque es factible identificar al menos dos segmentos distintos: los May (tres varones) y los Pat (cinco varones). Las mujeres, al estar casadas con alguno de los hombres con dichos patronímicos, pertenecían a la misma unidad de filiación.

Por su parte, en la jurisdicción de Bokobá, la estancia Mukuyche registró siete varones con dos apelativos distintos (Chac y Aké). La posibilidad de que se tratara de individuos pertenecientes a una misma unidad de filiación se refuerza con el hecho de que los indios portadores de tales patronímicos tenían un origen común. En contraste, la estancia Auatbach, ubicada en la jurisdicción de Izamal, fue conformada por más grupos que en las fincas anteriores, al tiempo que presentaba una mayor concentración de población, pues llegó a sumar en 1803 un total de 71 residentes mayores de 14 años. Incluso en esta densidad se reconoce la reagrupación de una buena parte de los indios de acuerdo con los principios básicos del ch'ibal, aunque en menor proporción que en las fincas de Cacalchén y Bokobá.

Como hemos visto anteriormente, la jurisdicción de Zitilpech fue una zona receptora de migrantes y la conformación de las fincas rurales quedó lejos de ser regular en cuanto a la distribución de los patronímicos. Un ejemplo basta para mostrar esta variación: la estancia Kantochen, donde entre sus 24 residentes difícilmente encontramos a más de dos indios o indias compartiendo el mismo apelativo.

No obstante, el panorama general que nos brinda la muestra elegida para este apartado nos permite plantear que existió una tendencia de los indios a mantener en sus nuevos lugares de residencia los principios que regían algunas de las formas de organización de los mayas. Se ha abordado ya la manera en que los ch'ibales y sus unidades 


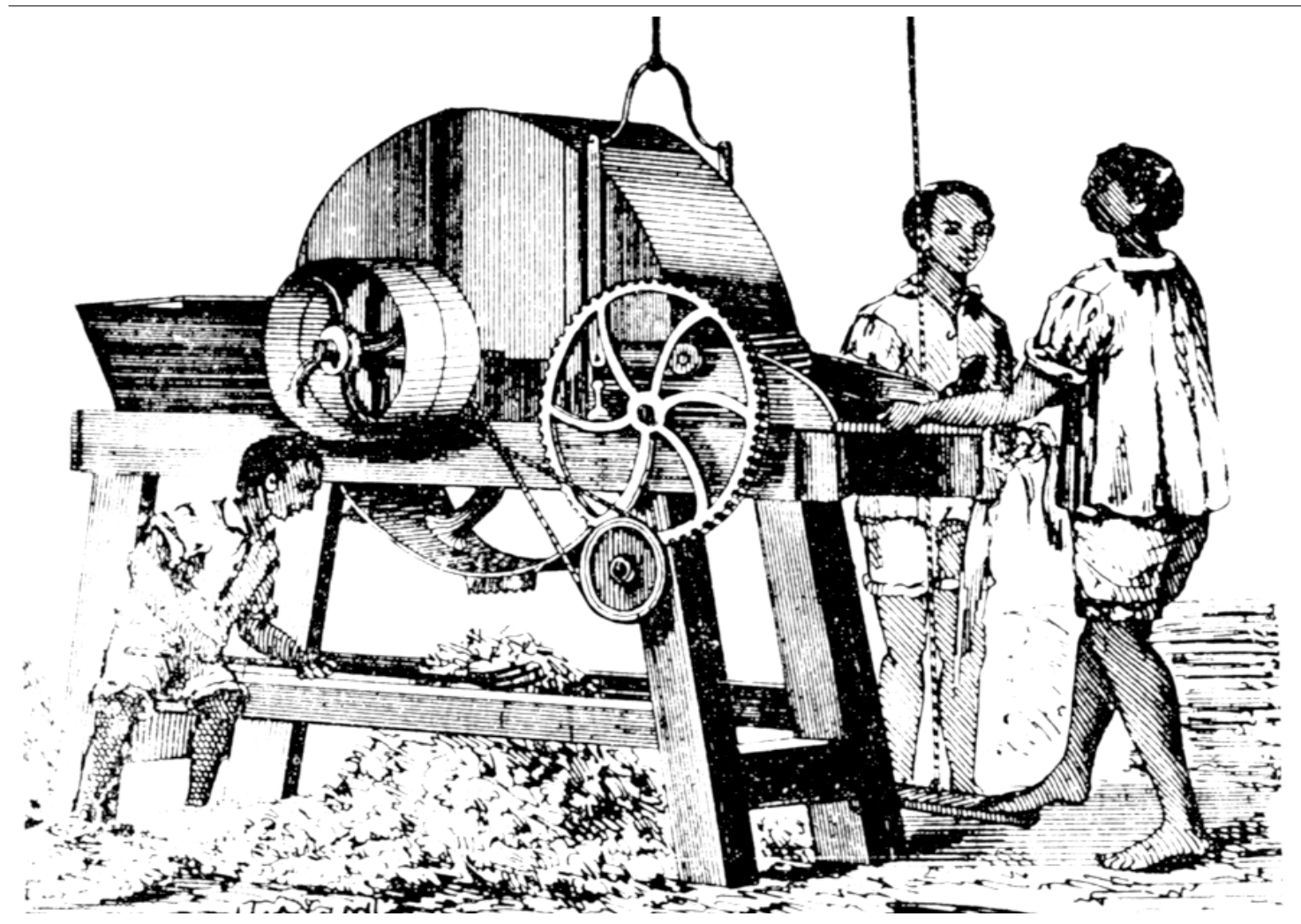

Juanes Patrullo, La raspadora (tomada de El repertorio pintoresco, Mérida, 1863).

fueron un elemento importante del paisaje político de los pueblos de indios. Por lo tanto, es posible que gran parte de la migración colectiva estuviera relacionada con los conflictos políticos internos y el manejo de recursos estratégicos, que obligaron a los ch'ibales — como células de organización- a trasladarse a otros lugares en busca de su sobrevivencia como grupo.

\section{CONSIDERACIONES FINALES}

Los chibales de la época colonial nos presentan una unidad de organización que ejemplifica claramente las características asignadas a los sistemas de linajes segmentarios. De acuerdo con este criterio, casi por definición, los linajes patrilineales tienden a subdividirse en unidades más pequeñas en las que existen fuerzas políticas con relativa autonomía, cuyas relaciones se definen en función de la presencia o ausencia de una fuerza política externa, creando alianzas o disolviéndolas en determinados contextos y situaciones políticas.

Así, los chibales conformaban un elemento de relevante importancia en el paisaje sociopolítico de los mayas coloniales. Los linajes y sus segmentos actuaban como unidades políticas al interior de otra célula de organización mayor, que en la época colonial quedó conformada como la república de indios. Sin embargo, en el siglo XVIII esta última entró en crisis, y la migración a las estancias ganaderas brindó la posibilidad a los segmentos de linaje de refuncionalizar en un nuevo espacio las relaciones que les daban sustento y perpetuarse como unidades de organización.

Este proceso estuvo influido por una serie de factores internos y externos, tales como el fraccionamiento de las tierras de los ch'ibales dominantes, la creciente tendencia hacia la privatización de tierras, así como una pérdida constante del control de la mano de obra de los macehuales. Este hecho llevó a la descomposición paulatina de la 
estratificación tradicional, en donde el sector de la población que había tenido en sus manos por largo tiempo el manejo de los recursos procuró conservar y reproducir determinadas relaciones que mantuvieran su posición en la organización política, hasta ese momento vigente.

En este sentido, la migración a las estancias parece haber sido una de las estrategias que los mayas adoptaron para tener acceso de manera alternativa a los recursos que garantizaran su subsistencia, llevando, inevitablemente, a un cambio en las relaciones de poder y las formas de articulación política entre la población maya colonial. No obstante, como hemos visto, esa transformación no vulneró las formas básicas de organización sociopolítica de los mayas coloniales. En nuestro tránsito por ciertas estancias del espacio colonial vimos la forma como algunos de los indios que migraron reprodujeron en los lugares de destino los principios de la unidad del ch'ibal.

Las estancias proporcionaron el espacio idóneo para la reproducción de las formas de organización que estaban en la médula de las relaciones sociales de los mayas. Es posible que los conflictos que se vivieron al interior de las repúblicas de indios hayan creado un faccionalismo interno, dando como resultado que los grupos menores que desempeñaban el papel de organizadores de la vida política de los pueblos — como los ch'ibales - se hayan escindido de la unidad política mayor, optando algunos de ellos por migrar a las estancias. Esto significó que los cambios que se experimentaron a nivel del pueblo o $c a h$, no implicaron que todo el universo de la organización sociopolítica de los mayas se estuviera transformando, sino que el principio de segmentación inherente a los linajes parentales nos permite plantear que, a pesar de la migración, existió una tendencia de los mayas a mantener tales unidades de organización.

\section{Siglas y bibliografía}

AGN: Archivo General de la Nación.

Calepino de Motul. Diccionario maya-español, 1995, Ramón Arzápalo Marín (ed.), 3 vols., UNAM, México.

Blanco, Cristina, 2000, Las migraciones contemporáneas, Alianza Editorial, Madrid.
Bracamonte y Sosa, Pedro, 200o, Jurisdicción y tenencia de la tierra entre los mayas de la provincia de Yucatán, informe de investigación, Mérida, Yucatán.

—_ y Gabriela Solís Robleda, 1996, Espacios mayas de autonomía. El pacto colonial en Yucatán, Universidad Autónoma del Estado de Yucatán, Mérida.

Diccionario Maya Cordomex, 1980, Alfredo Barrera Vázquez (dir.), Ediciones Cordomex, Mérida.

Dumont, Louis, 1975, Introducción a dos teorías de la antropología social, Editorial Anagrama, México.

Evans Pritchard, E. E., 1977, Los Nuer, Anagrama, Barcelona. Farriss, Nancy, 1978, "Nucleation Versus Dispersal: The Dynamics of Population Movement in Colonial Yucatán", Hispanic American Historical Review, vol. 58, núm. 2, 1978.

__ 1992, La sociedad maya bajo el dominio colonial, Alianza, Madrid.

Fox, Robin, 1967, Sistemas de parentesco y matrimonio, Alianza Universidad, Madrid.

García Bernal, Manuela Cristina, 1972, La sociedad de Yucatán, 1700-1750, Escuela de Estudios Hispano-Americanos de Sevilla, Sevilla.

— 1994, "Desarrollos indígena y ganadero en Yucatán", Historia Mexicana, vol. XLIII, núm. 3, enero-marzo, El Colegio de México, México.

Middleton, John y Davis Tait, 1968, "The Lineage and de Lineage System”, en Paul Bohannan (ed.), Kingship and Social Organization, The Natural History Press, Nueva York.

Okoshi Harada, Tsubasa, 1992, Los canules: análisis etnohistórico del Códice de Calkiní, tesis de doctorado, UNAM, México.

— 2000 , "Los Xiu del siglo XVI: una lectura de dos textos mayas coloniales", Mesoamérica, año 21, núm. 39, junio, Plumsock Mesoamerican Studies/CIRMA, Antigua.

Patch, Robert, 1993, Maya and Spaniard in Yucatán, 16481812, Stanford University Press, Stanford, California.

Peniche Moreno, Paola, 2002, La diáspora indígena a las estancias yucatecas del siglo XIII, tesis de maestría en antropología social, CIESAS, México.

Restall, Mathew, 1997, The Maya World. Yucatec Culture and Society, 1550-1850, Stanford University Press, Stanford, California.

Reyes García, Luis, 1996, "El término calpulli en documentos del siglo XVI", en Luis Reyes García et al., Documentos nahuas de la ciudad de México del siglo XVI, CIESAS, México.

Roys, Ralph L., 1943, The Indian Background of Colonial Yucatán, Carnegie Institution of Washington, Washington.

Solís Robleda, Gabriela, 2000, Bajo el signo de la compulsión. El trabajo forzoso indígena en el sistema colonial yucateco, 1540-1730, tesis doctoral, UNAM, México. 